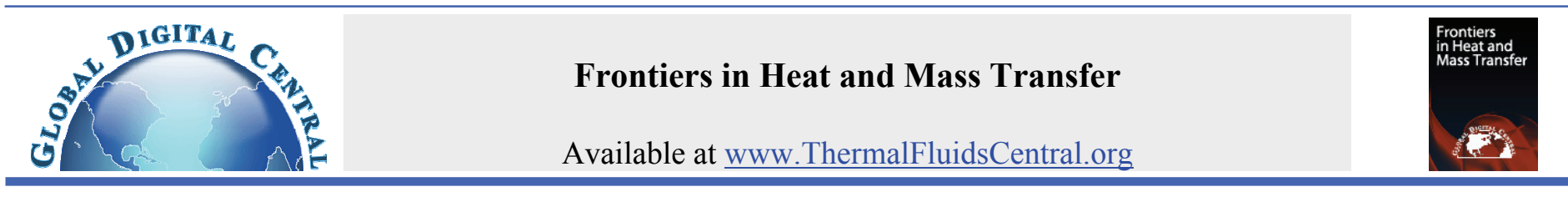

\title{
HEAT GENERATION EFFECTS ON NATURAL CONVECTION IN POROUS CAVITY WITH DIFFERENT WALLS TEMPERATURE
}

\author{
Majid Tahmasebi Kohyani ${ }^{\mathrm{a}}$, Behzad Ghasemi ${ }^{\mathrm{a}}$, Ahmad Pasandideh Fard ${ }^{\mathrm{b}}$ \\ ${ }^{a}$ Department of Mechanical Engineering, Shahrekord University, Shahrekord, Iran \\ ${ }^{b}$ Mohajer center, Technical and Vocational University, Esfahan, Iran
}

\begin{abstract}
Natural convection heat transfer in a square cavity with a porous medium subjected to a uniform energy generation per unit volume is studied numerically in this paper. Temperature of the vertical walls is not equal but it is constant . There are two effective parameters in this condition that appear in the nondimensionalized equations and they are functions of temperature difference between hot and cold walls and energy generation in the porous medium. Nondimensionalized governing equations are obtained based on the Darcy model. a control volume approach is used for solving these equations. The effects of the variation of two governing parameters are investigated on the heat transfer rate, fluid flow and isotherms.
\end{abstract}

Key words: porous medium, natural convection, heat generation, cavity

\section{INTRODUCTION}

Porous media and the corresponding heat transfer phenomena has an important place in the industry. This importance has motivated many researchers to investigate the effective parameters on heat transfer in the porous media. Using porous media in the industry has many applications such as underground heat exchangers for energy saving, energy recovery and control of reactors temperatures, cooling electronic devices, thermal insulations, geothermal energy, etc. These and more applications are found in books of publishers such as Nield and Bejan (2006), Ingham et al. (2004), Ingham and Pop (2005) and Vafai (2000).

Heat transfer in a porous medium with different boundary conditions is one of important subjects considered in the literature. Anderson and Larit (1986) studied natural convection in a rectangular cavity with uniform wall temperatures where the vertical walls were cold, the bottom wall hot, and the upper wall adiabatic. Kimura and Bejan (1985) studied natural convection in rectangular chamber numerically, with cold temperature for bottom wall and constant heat flux for one of vertical wall. Pop and Saeid (2004) registered an article in porous medium field and showed increasing of Ra decreases time to achieve to constant Nuselt number. Chao and Ozoe (1983) analyzed natural convection in inclined cavity that half of bottom wall was adiabatic and second half had constant heat flux, and upper wall was cold. Granzarolli and Milanez (1995) reported results for cavity with cold temperature at vertical walls and constant heat flux at bottom side. Saeid (2007) presented a problem with both conduction and natural convection in porous medium that enclosed with finite thickness walls. Saeid and Pop (2005) have studied the natural convection in vertical porous medium with two heat source. They reported effects of $\mathrm{Ra}, \mathrm{Pe}$ (Peclet number) and distance between heat sources on heat transfer. Chamkha et al. (2002) studied a porous cavity that is exposed to radiation heat transfer. Grosan et al. (2009) investigated the effects of magnetic field and heat generation on porous cavity and they presented results for effect of $\mathrm{Ra}$ and Ha number in heat transfer of cavity. Ha is a parameter that depends to magnetic field intensity.
Mealey and Merkin (2009) considered heat generation in porous mediums. They have shown that Edi vortexes created in center of vertical walls at large Ra number.

However, a very little works has been done for the case of heat generation effect on porous medium. The aim of this paper is to design different parameters and perform a extensive study in conditions that in addition to heat generation in porous cavity, The temperature of vertical walls is different.

\section{GOVERNING EQUATIONS}

A schematic diagram of simple porous medium with different boundary conditions and uniform heat generation per unit volume is shown in Fig. 1. The vertical walls are at constant temperature $T_{c}$ and $T_{h}$ and horizontal walls are adiabatic. In the porous medium Darcy's law and Boussinesq approximation have been used and also fluid and solid are in thermodynamics equilibrium. The viscous drag and inertia terms in the governing equations are neglected which are valid assumptions for low Darcy and particle Reynolds numbers. With these assumptions, The Continuity, Darcy and energy equation for steady two dimensional flow in an isotropic and homogenous porous medium are:

$$
\begin{aligned}
& \frac{\partial u}{\partial x}+\frac{\partial v}{\partial y}=0 \\
& \frac{\partial u}{\partial y}-\frac{\partial v}{\partial x}=-\frac{g K \beta}{v} \frac{\partial T_{p}}{\partial x} \\
& u \frac{\partial T_{p}}{\partial x}+v \frac{\partial T_{p}}{\partial y}=\alpha\left(\frac{\partial^{2} T_{p}}{\partial x^{2}}+\frac{\partial^{2} T_{p}}{\partial y^{2}}\right)+\frac{q^{\prime \prime \prime}}{\rho C_{P}}
\end{aligned}
$$

\footnotetext{
*Corresponding author.Email: matkoh@yahoo.com
} 
Where the subscript $\mathrm{p}$ is for porous. These equations can be written in term of stream function $\psi$ (defined as $u=\frac{\partial \psi}{\partial y}$ and $v=-\frac{\partial \psi}{\partial x}$ ).

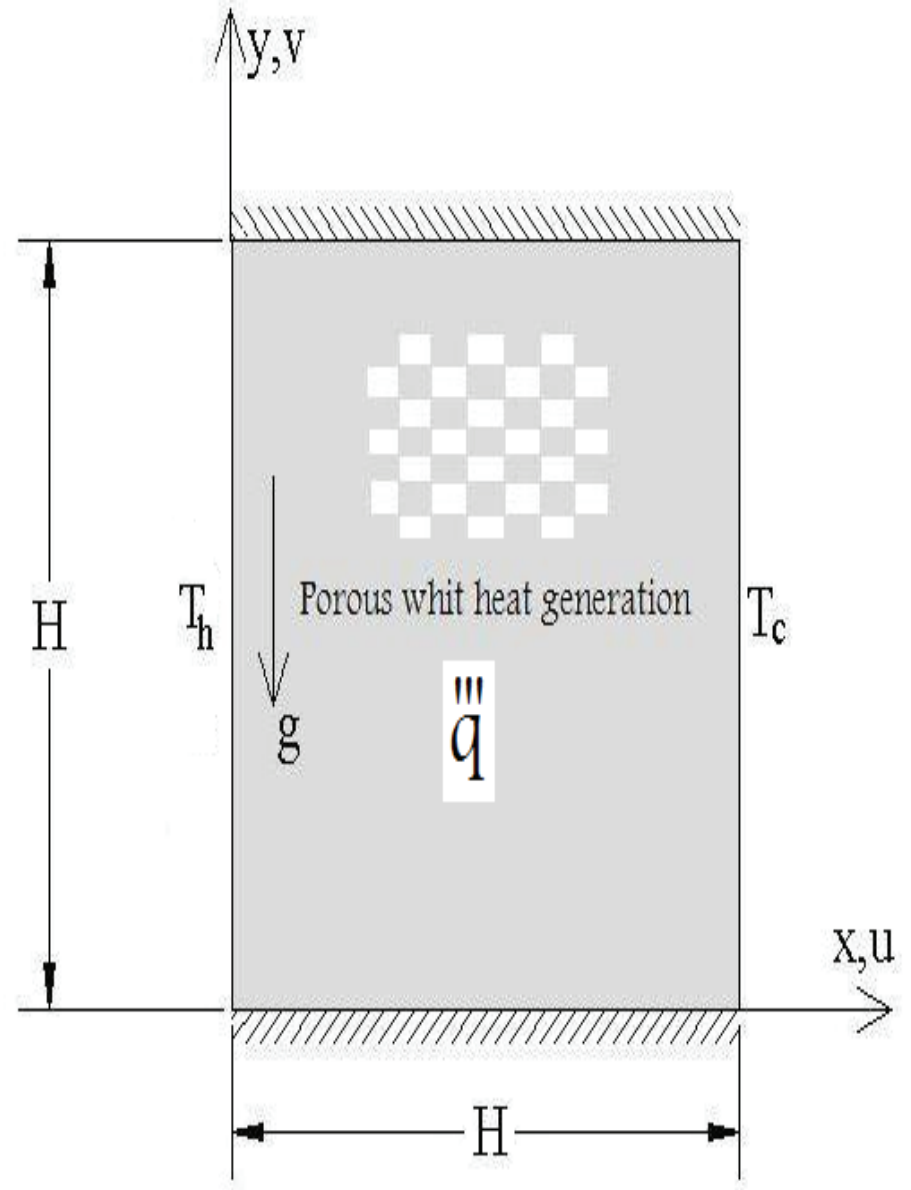

Fig. 1 Geometry and boundary condition of the problem and coordinate system.

By defining nondimensional variables as:

$\Psi=\frac{\psi}{\alpha} ; \theta=\frac{T-T_{c}}{T_{h}-T_{c}} ; X=\frac{x}{H} ; Y=\frac{y}{H}$

governing equations [1-3] can be rewritten as :

$\frac{\partial^{2} \Psi}{\partial X^{2}}+\frac{\partial^{2} \Psi}{\partial Y^{2}}=-R a \frac{\partial \theta_{p}}{\partial X}$

$\frac{\partial \Psi}{\partial Y} \frac{\partial \theta_{p}}{\partial X}-\frac{\partial \Psi}{\partial X} \frac{\partial \theta_{p}}{\partial Y}=\frac{\partial^{2} \theta_{p}}{\partial X^{2}}+\frac{\partial^{2} \theta_{p}}{\partial Y^{2}}+\lambda_{p}$

where $\mathrm{Ra}$ is the Rayligh number defined as:

$$
\mathrm{Ra}=\left(\mathrm{g} \cdot \beta \cdot \mathrm{K} \cdot\left(T_{h}-T_{c}\right) \cdot \mathrm{H}\right) /(v \alpha)
$$

and $\lambda \mathrm{p}$ is a parameter that is related to heat generation in porous medium and defined as :

$$
\lambda_{p}=\frac{q^{\prime \prime \prime} H^{2}}{k\left(T_{h}-T_{c}\right)}
$$

The value of non-dimensional stream function are zero on cavity's walls:

$$
\Psi(0, Y)=\Psi(1, Y)=\Psi(X, 0)=\Psi(X, 1)=0
$$

and boundary conditions for non-dimensional temperature are:

$$
\begin{aligned}
& \theta(0, Y)=1, \theta(1, Y)=0 \\
& \frac{\partial \theta(X, 0)}{\partial Y}=\frac{\partial \theta(X, 1)}{\partial Y}=0
\end{aligned}
$$

The physical quantities in this problem are local and average Nusselt number that defined in vertical walls as:

$$
\overline{N u}=\int_{0}^{1} N u d y \quad ; \quad N u=-\frac{\partial \theta}{\partial X}
$$

\section{NUMERICAL METHOD}

Equations (5) and (6), with boundary conditions Eqs. (9-11), are integrated numerically by finite volume method (Patankar, 1966). The power law scheme is used for convection - diffusion terms in Eq. (6) and central difference approximation is used for diffusion terms of Eqs. (5) and (6).

For having accurate answers, it's need to use an appropriate mesh scheme for geometry and boundary conditions. For mesh generation, different techniques can be used (Hoffmann, 1993).

In present work algebraic method is used for mesh generation. But near of the boundaries, grid scheme is finer than central region because gradient of parameters in boundaries are bigger than central region. A used sample mesh scheme is shown in Fig. 2. For studying effect of grid sheme on results, variation of one of important parameters $\Psi_{\max }$ with mesh size has been shown in Fig. 3.

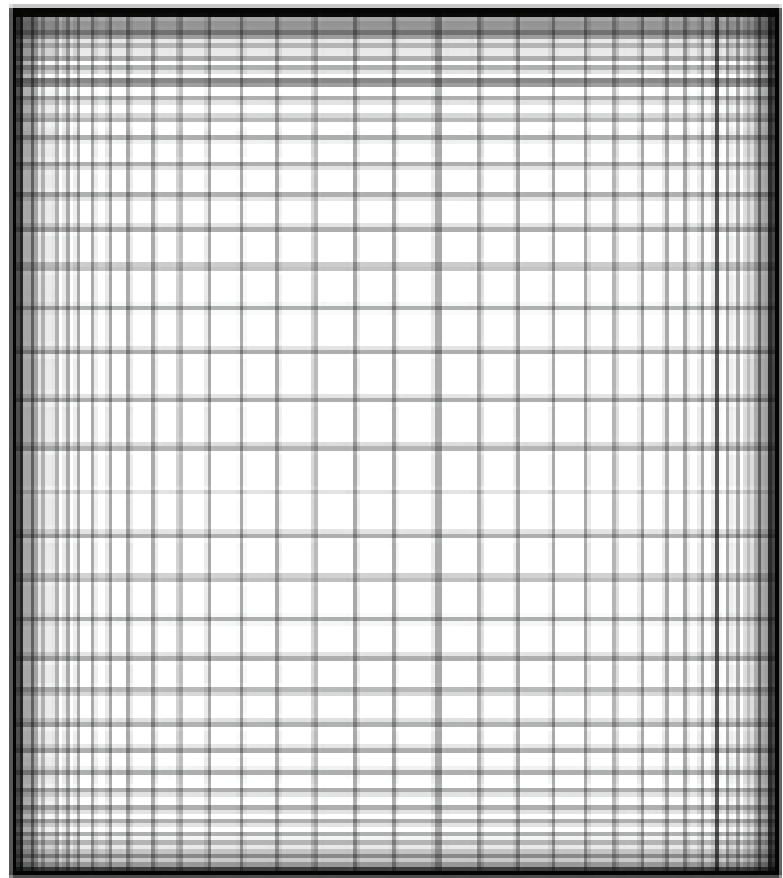

Fig. 2 Schematic of the mesh 


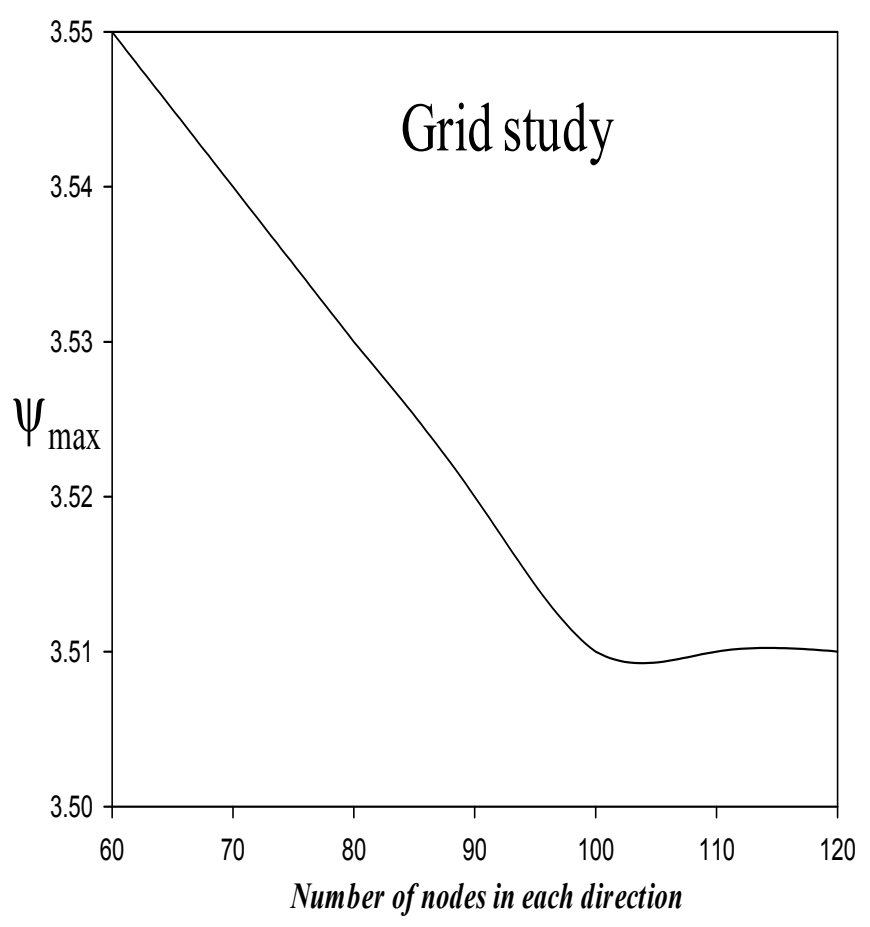

Fig. 3 Negligible variation of the $\Psi_{\max }$ with increase of number of nodes for $\mathrm{Ra}=1000$ and equal temperature of vertical walls .

Fig. 2 shows that variation of $\Psi_{\max }$ with mesh size is negligible for mesh bigger than $101 \times 101$. Therefore for appropriate and accurate solving, a grid size with $101 \times 101$ mesh is used. It should be said that not only for Fig. 3 that is depicted for condition that temperature of vertical walls is equal and in this case in non-dimensional equations only $\mathrm{Ra}$ is appeared, But also for non-equal vertical walls temperature for different $\mathrm{Ra}$ and $\lambda \mathrm{p}$ after $101 \times 101$ grids variation of basic parameters such as $\Psi_{\max }$ and $\theta_{\max }$ is negligible. The resulting algebraic equations, have been solved by line by line iteration using tri-diagonal matrix algorithm. Iteration of process is terminated under the following condition:

$\left[\sum\left|\varphi_{i, j}^{n}-\varphi_{i, j}^{n-1}\right| / \sum_{i, j}\left|\varphi_{i, j}^{n}\right|\right] \leq 10^{-8}$

\section{VALIDATION}

To ensure of the accuracy of this program, the results have been compared in special case in presented work by Grosan et al. (2009). This comparison is carried out in conditions that vertical walls temperature are equal, the horizontal walls are adiabatic and $\mathrm{Ra}=1000$ and there is no magnetic field and heat is generating per volume unit in cavity. Stream lines and isotherm lines are presented for both works. in Figs. 4, a and b respectively. This comparison shows that stream lines and isotherm lines are so consistent. Numerical results for parameters $\Psi_{\max }$ and $\theta_{\max }$ are also given in Table 1 .

Small difference between the results is due to different approximation and different convergence criteria during the solving process. After evaluation of applying computer code, results have been studied in two different cases, with $\mathrm{Ra}$ range $(10 \leq \mathrm{Ra} \leq 5000)$ and $\lambda \mathrm{p}$ range $(10 \leq \lambda \mathrm{p} \leq$ 100).

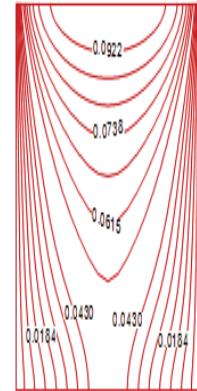

4.a this work
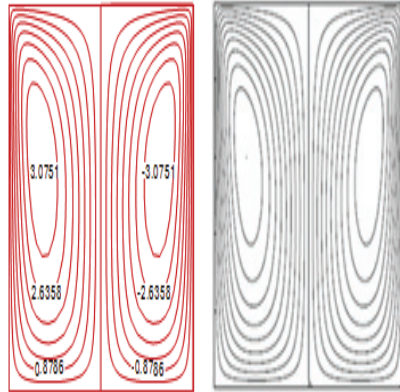

4.b. Grosan et al.(2009)
Fig. 4 Compartion of the stream lines and isotherm lines for this work and presented article by Grosan et al. (2009) at $\mathrm{Ra}=1000$ and $\mathrm{Ha}=0$.

Table $1 \Psi_{\max }$ and $\theta_{\max }$ in porous for this work and presented article by Grosan et al. (2009) at $\mathrm{Ra}=1000$ and $\mathrm{Ha}=0$.

\begin{tabular}{ccc}
\hline $\mathrm{Ra}=1000$ & $\Psi_{\max }$ & $\theta_{\max }$ \\
\hline Grosan et al. & 3.53 & 0.097 \\
This work & 3.51 & 0.098 \\
\hline
\end{tabular}

\section{DISCUSSION AND RESULTS}

\subsection{Effects of Ra variation at constant $\lambda p$}

In first case at constant $\lambda p$, effect of Ra variations has been studied on the thermal fields, and circulation of fluid in the porous enclosure. Isotherm lines and stream lines are presented in fig (5) and also variation of $\theta_{\max }$ and $\Psi_{\max }$ with Ra is illustrated in fig (6).

It can be seen from figs ( 5 and 6 ) that by increasing of $\mathrm{Ra}$, at constant $\lambda \mathrm{p}, \theta_{\max }$ decreases and $\Psi_{\max }$ increases. This is due to increasing of convection heat transfer in porous enclosure that causes more cooling and increase of power of vortexes. For low $\mathrm{Ra}, \mathrm{Ra}=10$, at $\lambda \mathrm{p}=100$ two symmetry and similar vortexes has been created.

However, by increasing of $\mathrm{Ra}$ the power of vortexes increases but counter clockwise vortexes are limited to top and left of cavity. Also it can be seen that isotherm lines are changed from almost elliptic lines to horizontal lines as shown in fig(5) that shows conversion of conduction heat transfer to convection.

Variation of the average Nuselt , $\overline{\mathrm{Nu}}$, has been illustrated with $\mathrm{Ra}$ in cold (cw) and hot wall (hw) in fig (7). By attention in fig (7) three points is considerable:

1. Increasing of average $\mathrm{Nu}$ in vertical boundries with increasing of Ra.

2. The average $\mathrm{Nu}$ is negative, $N u=-\frac{\partial \theta}{\partial X} \leq 0$, at low $\mathrm{Ra}$ that is due to the over coming of the thermal field in the porous cavity to temperature gradient between vertical boundaries that leads to exiting of the heat flux from high temperature boundary such as low temperature boundary.

3. The difference between values of $\mathrm{Nu}$ in cold and hot wall with increasing of $\mathrm{Ra}$ is almost constant and equals to $\lambda \mathrm{p}$. Therefore energy balances is as: $\overline{\mathrm{Nu}}_{\mathrm{Cw}}-\overline{\mathrm{Nu}}_{\mathrm{Hw}}=\lambda_{\mathrm{p}} \times \mathrm{H}^{2}$. 

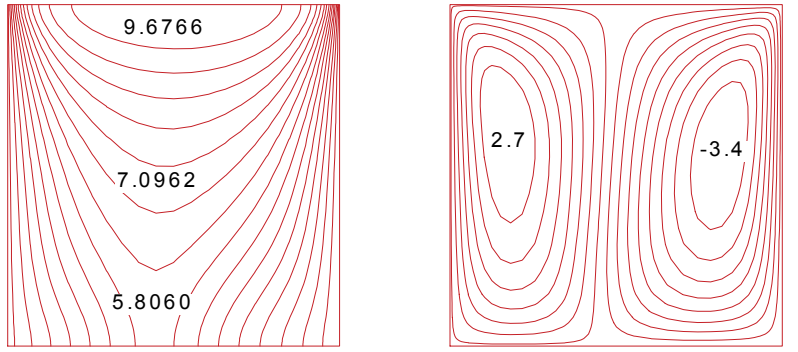

$\Psi_{\max }=3.17, \Psi_{\min }=-3.84, \theta_{\max }=10.32$

(a) $\mathrm{Ra}=10$
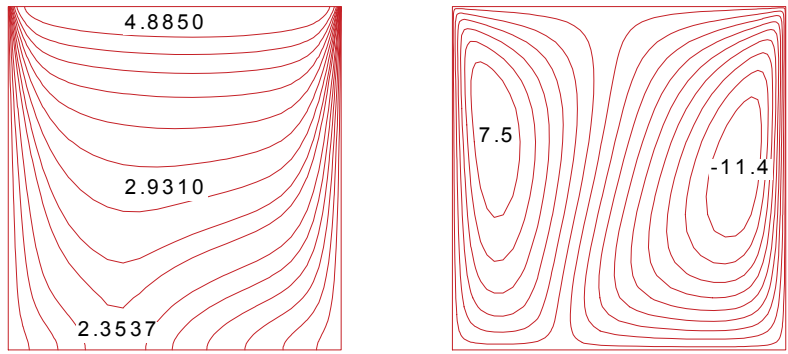

$\Psi_{\text {max }}=8.86, \Psi_{\text {min }}=-12.71, \theta_{\text {max }}=5.21$

(b) $\mathrm{Ra}=100$
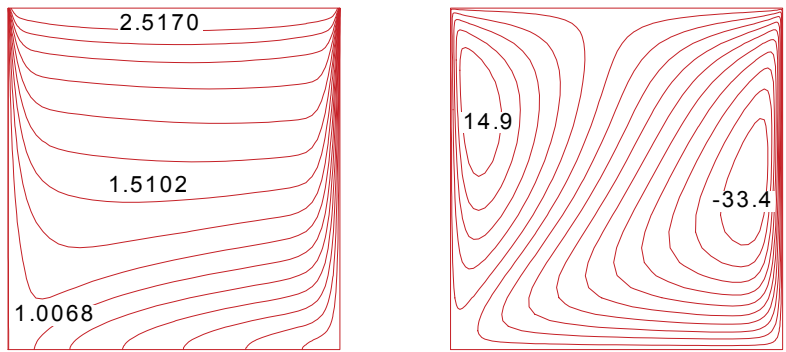

$\Psi_{\text {max }}=18.39, \Psi_{\text {min }}=-36.85, \theta_{\max }=2.68$

(c) $\mathrm{Ra}=1000$
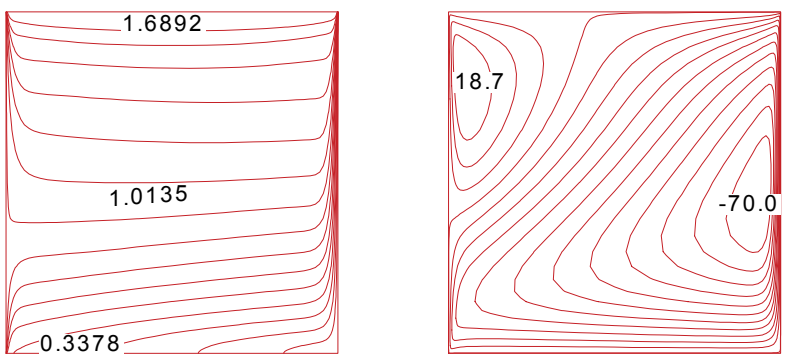

$\Psi_{\text {max }}=25.03, \Psi_{\text {min }}=-76.31, \theta_{\text {max }}=1.81$

(d) $\mathrm{Ra}=5000$

Fig. 5 Streamlines (on the right), and isotherms (on the left) in simple porous cavity with heat generation at $\lambda_{p}=100$ for temperature of vertical wall $T_{h}$ and $T_{c}$

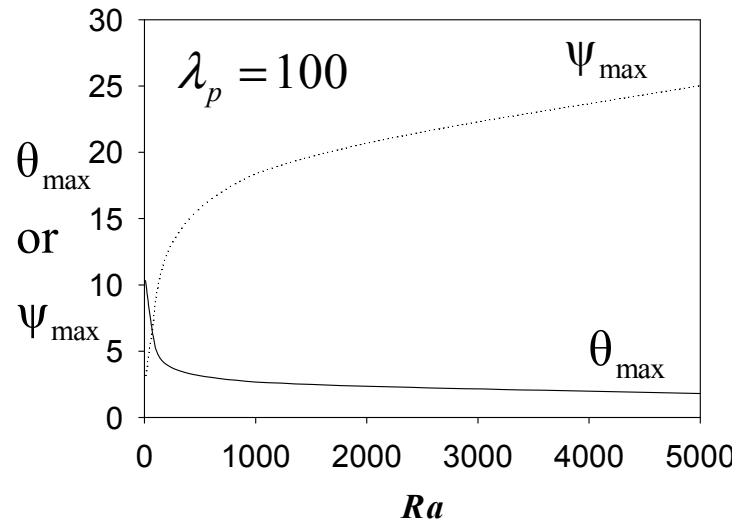

Fig. 6 Variation of $\theta_{\max }$ and $\psi_{\max }$ with $\mathrm{Ra}$ at $\lambda_{p}=100$

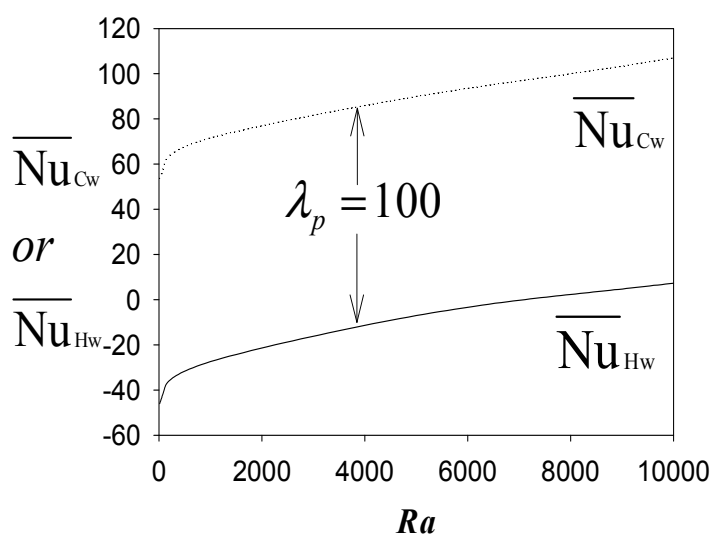

Fig. 7 Variation of $\overline{\mathrm{Nu}}$ in cold $(\mathrm{Cw})$ and hot $(\mathrm{Hw})$ walls with $\mathrm{Ra}$ at

$$
\lambda_{p}=100
$$

\subsection{Effects of $\lambda p$ variations at constant Ra}

In the second case effect of variation of $\lambda \mathrm{p}$ at constant $\mathrm{Ra}$ has been investigated. Schematic of isotherm and stream lines are shown in Fig (8) and variation of $\theta_{\max }$ and $\psi_{\max }$ are presented in fig (9) for $\mathrm{Ra}=1000$.

As indicated in figs 8 and 9 , increasing of $\lambda p$ that is equal to increasing of heat generation, causes heating of enclosure. Therefore power of vortexes increases and it can be seen that $\theta_{\max }$ and $\psi_{\max }$ increase too. By attention in fig 8 , it is clear that in opposite of fig 5 , that increasing of $\mathrm{Ra}$ at $\lambda \mathrm{p}=100$ reduces area of positive vortexes and finally delete them, Increasing of $\lambda \mathrm{p}$ at constant $\mathrm{Ra}$ creates positive vortexes and increase their area.

Also it can be seen in fig 8 that by increasing of $\lambda$, isotherm lines nearly become horizontal that shows that power of convection heat transfer is increased. Variation of average $\mathrm{Nu}$, with $\lambda \mathrm{p}$, on cold and hot walls is shown in fig 10 for $\mathrm{Ra}=1000$. As indicated in fig 10 , however, with increasing of $\lambda \mathrm{p}, \mathrm{Nu}$ increases in vertical boundaries but difference between $\mathrm{Nu}$ in cold and hot walls is equal to $\lambda p$ that shows energy balance in cavity. It is important to notice that negative $\mathrm{Nu}$ on hot wall is due to emersion of heat of this wall that is because of the thermal field in the cavity. 


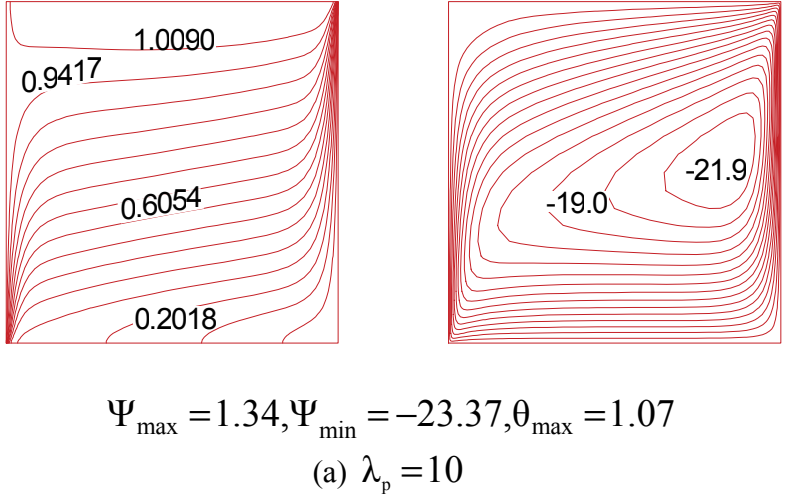

\begin{tabular}{|c|}
\hline 2.5170 \\
\hline 1.8458 \\
1.5102 \\
1.0068 \\
\hline
\end{tabular}

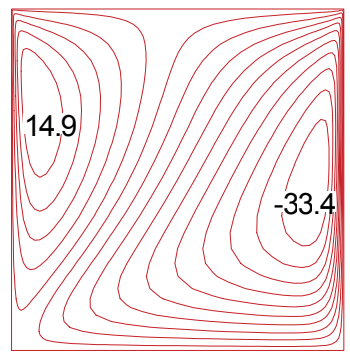

$$
\Psi_{\text {max }}=18.39, \Psi_{\text {min }}=-36.85, \theta_{\max }=2.68
$$

(a) $\lambda_{\mathrm{p}}=100$

\begin{tabular}{|c|}
6.5414 \\
4.3610 \\
\hline 3.0527 \\
\hline 2.1805 \\
\hline
\end{tabular}

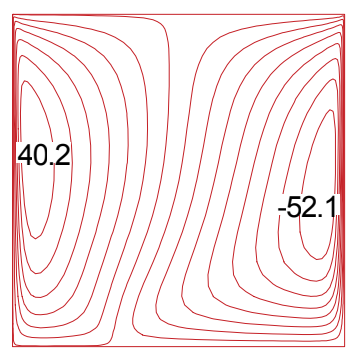

$\Psi_{\max }=46.82, \Psi_{\min }=-58.69, \theta_{\max }=6.97$

(c) $\lambda_{\mathrm{p}}=500$

\begin{tabular}{c}
9.4667 \\
\hline 5.6800 \\
\hline 4.4178 \\
\hline 3.1556 \\
\hline
\end{tabular}

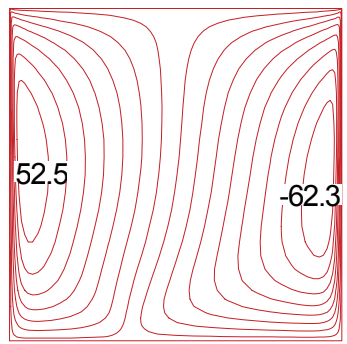

$$
\Psi_{\max }=60.71, \Psi_{\min }=-70.52, \theta_{\max }=10.09
$$

(d) $\lambda_{\mathrm{p}}=1000$

Fig. 8 Streamlines (on the right), and isotherms (on the left) in simple porous cavity with heat generation at $\mathrm{Ra}=1000$ for temperature of vertical wall $T_{h}$ and $T_{c}$

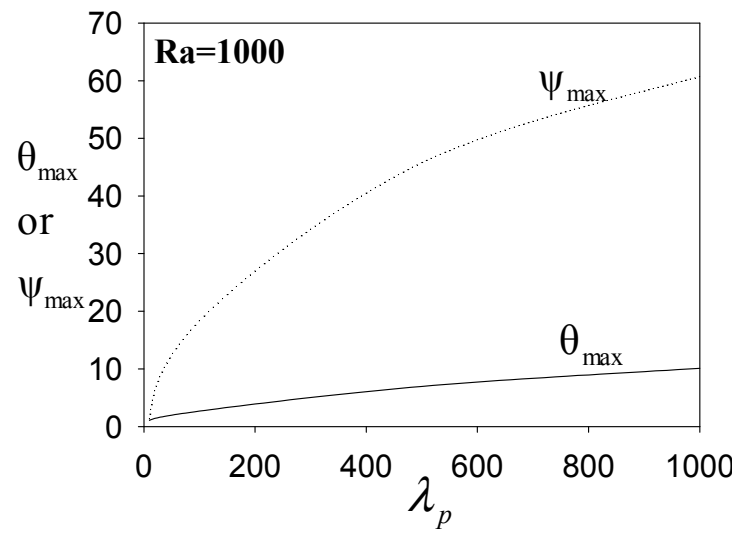

Fig. 9 Vareation of $\theta_{\max }$ and $\psi_{\max }$ with $\lambda_{\mathrm{p}}$ at $\mathrm{Ra}=1000$

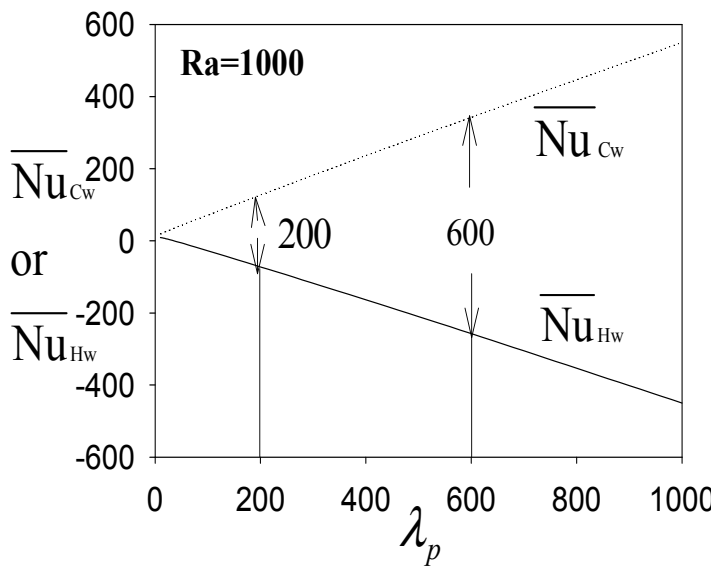

Fig. 10 Variation of $\overline{\mathrm{Nu}}$ in cold $(\mathrm{Cw})$ and hot (Hw)wall with $\lambda_{\mathrm{p}}$ at $\mathrm{Ra}=1000$

\section{CONCLUSIONS}

Natural convection heat transfer in cavity containing the porous medium with heat generation per unit volume is considered for numerical investigation. The governing parameters are $\mathrm{Ra}$ and $\lambda \mathrm{p}, \mathrm{Ra}$ is function of temprature difference of cold and hot boundries. Parameter $\lambda p$ is related to heat generation in cavity. The non-dimensional forms of the continuity, Darcy and energy equations are solved numerically. Parametric study is carried out and the results are presented to show the effect of these parameters on heat transfer and fluid flow characteristics. It is found that by increasing $\mathrm{Ra}$ at constant $\lambda \mathrm{p}$, the power of vortexes increases but maximum temprature of cavity, $\theta_{\max }$ decreases. Also numerical results indicated that by increasing of $\lambda p$ at constant $R a$, both of $\theta_{\max }$ and power of vortexes increases. also, it is shown that by increasing $\mathrm{Ra}$ or $\lambda \mathrm{p}$ the $\overline{\mathrm{Nu}}$ increases in cold and hot bondries and energy balance is indefeasible as:

$\overline{\mathrm{Nu}}_{\mathrm{Cw}}-\overline{\mathrm{Nu}}_{\mathrm{Hw}}=\lambda_{\mathrm{p}} \times \mathrm{H}^{2}$

\section{NOMENCLATURE}

$\mathrm{H} \quad$ height of cavity

g gravitational acceleration

R Rayleigh number

$\lambda_{\mathrm{p}} \quad$ non-dimensional heat generation parameter

$\overline{\mathrm{Nu}} \quad$ average Nusselt number 
$\mathrm{N} \quad$ local nusselt number

$\mathrm{K}$ permeability of the porous medium

$\mathrm{k} \quad$ effective thermal conductivity of the porous medium

$q^{\prime \prime \prime} \quad$ heat generation per unit volume in porous medium

$\mathrm{T}_{\mathrm{p}} \quad$ temperature of porous medium

$\mathrm{T}_{\mathrm{h}}, \mathrm{T}_{\mathrm{c}} \quad$ temperature of cold and hot wall

$\mathrm{U}, \mathrm{V} \quad$ axial and radial velocities

$\mathrm{X}, \mathrm{Y} \quad$ non-dimensional coordinates

Greek Symbols

$\begin{array}{ll}\alpha & \text { thermal diffusivity } \\ \theta & \text { non-dimensional temperature } \\ v & \text { kinematic viscosity } \\ \beta & \text { thermal expansion coefficient } \\ \psi & \text { stream function } \\ \Psi & \text { non-dimensional stream function }\end{array}$

\section{REFERENCES}

Nield, D. A. and Bejan, A. ,2006, Convection in porous media, Third Edition, USA.

Ingham, D. B., Bejan, A., Mamut, E. and Pop, I. ,2004, Emerging Technologies Techniques in porous media, Kluwer Academic, Dordrecht.

http://dx.doi.org/10.1007/978-94-007-0971-3

Ingham, D. B. and Pop, I. Vol.1. ,2002, . Vol.2. ,2005, Transport phenomena in porous media, Pergamon, Oxford.

Vafai, K. ,2000, Hand Book of Porous Media, New York.

Anderson, R. and Lauriat, G. ,1986, "The horizontal natural convection boundary layer regime in a closed cavity," in: proc. $8^{\text {th }}$, International Heat Transfer Conf. San Francisco, USA 98, pp. 1453 -1458.

Kimura, S. and Bejan, A. ,1985, "Natural convection in defferentially heatedcorner region," Phys. Fluids 28, pp. $2980-2989$.
Pop , I. and Saeid, N. H. ,2004, "Transient free convection in a square cavity filled with a porous medium," International J. Heat and Mass Transfer48, pp. 1917-1924.

Chao, P. and Ozoe, H. ,1983, "Laminar natural convection in an inclined rectangular box with lower surface half heaten and half insulated," ASME J. Heat and massTransfer105, pp. 425-432.

Granzarolli, M. M. and Milanez, L. F. ,1995, "Natural convection in rectangular enclosures heated from below and symmetrically cooled from the sides," International J. Heat Mass Transfer 388, pp. 1063 1073.

Saeid, N. H. ,2007, "Conjugate natural convection in a vertical porous layer sandwiched by finite thickness walls," International Heat Transfer Conf 34, pp. 200- 216.

http://dx.doi.org/10.1016/j.icheatmasstransfer.2006.11.003

Pop, I. and Saeid , N. H. ,2005, "Mixed convection from two thermal sourses in a vertical porous layer," International J. Heat and Mass Transfer 48 , pp. $4150-4160$.

Chamkha, A. j., Issa, C. and khanafer, K ,2002, "Natural convection from an inclined plate embedded in a variable porosity porous medium due to solar radiation," Int. J. Therm. Sci 41, pp.73-81.

\section{http://dx.doi.org/10.1016/S1290-0729(01)01305-9}

Grosan, T. C., Revnic, b., Pop, I., and Ingham, D.B., 2009, "Magnetic field and internal heat generation effects on the free convection in a rectangular cavity filled with a porous medium," International Journal of Heat and Mass Transfer 52, pp. 1525-1533.

http://dx.doi.org/10.1016/j.ijheatmasstransfer.2008.08.011

Mealey, L. R. and Merkin, J .H. ,2009, "Steady finite Rayleigh number convective flows in a porous medium with internal heat generation," International Journal of Thermal Sciences 48, pp. 1068-1080.

http://dx.doi.org/10.1016/j.ijthermalsci.2008.10.008

Patankar, S. V. ,1996, Computation of conduction and Duct Flow Heat Transfer, Innovative Research, Inc. USA.

Hoffmann, K. A. and Chiang, S. T. ,1993, Computational fluid dynamics for engineers, USA. 